\title{
Non-classical photon statistics for two-mode optical fields
}

\author{
B Arvind $\dagger \S$ and N Mukunda $\ddagger \|$ \\ $\dagger$ Department of Physics, Indian Institute of Science, Bangalore-560 012, India \\ $\ddagger$ Center for Theoretical Studies and Department of Physics, Indian Institute of Science, \\ Bangalore-560 012, India
}

\begin{abstract}
The non-classical feature of sub-Poissonian photon statistics is extended from one- to two-mode electromagnetic fields, incorporating the physically motivated property of invariance under passive unitary transformations. Applications to squeezed coherent states, squeezed thermal states, and superposition of coherent states are given. Dependences of extent of nonclassical behaviour on the independent squeezing parameters are graphically displayed.
\end{abstract}

\section{Introduction}

Non-classical properties and effects of radiation [1] have received considerable attention in the past two decades and continue to be an active area of research. Quadrature squeezing [2], sub-Poissonian photon statistics (SPS), and antibunching [3] of photons are three prominent and independent examples of such properties leading to measurable effects. Quadrature squeezing is related to the reduction of noise in one of the two quadrature components below the coherent state value, and has been both theoretically and experimentally studied for one-mode as well as multi-mode fields. Antibunching arises when the photon number distribution becomes sub-Poissonian leading to anticorrelation in the photons detected in a typical detection experiment. In all these cases, the diagonal coherent state description of the fields involved does not have a classical interpretation and hence no classical description can explain these effects.

The extension from one to two or more modes for the case of quadrature squeezing is non-trivial and leads to new physical effects $[4,5]$. The phenomenon of SPS has been formulated [6] and observed [7] primarily for one-mode situations. For situations involving two or more modes, in the existing literature, such properties are invariably studied for one of the modes or a predefined linear combination of the modes [8]. Such an analysis cannot be used to make any clear cut statement about the classical or non-classical nature of the field involved, because the linear combination of modes which may show SPS may in general be different from the mode chosen for the analysis. Another kind of generalization of SPS to two-mode fields has been suggested using a particular inequality involving the correlation between the two modes; however, this does not exhaust the possibilities available at the level of quadratic expressions in photon number [9]. This clearly indicates the need for a more satisfactory and comprehensive way of looking at non-classical statistics, for fields involving two or more modes.

$\S$ E-mail address: arvind@physics.iisc.ernet.in

\| Also at Jawaharlal Nehru Centre for Advanced Scientific Research, Jakkur Bangalore-560 064, India. 
Our aim in this paper is to develop a notion of SPS for two-mode fields which is intrinsically two-mode in character, can be used in an unambiguous way to make a statement about the classical or non-classical nature of the field, and has physically reasonable invariance properties. The group of linear, homogeneous, canonical transformations $S p(4, \Re)$, the symmetry group basic to the quantum mechanical description of the twomode field, naturally splits into two parts: the photon number conserving (maximal compact) passive subgroup $U(2)$, and the photon number non-conserving (non-compact) active part. The maximal compact subgroup $U(2)$, while acting on the Hilbert space of the twomode system through its unitary representation, is incapable of generating a non-classical (classical) state starting from a classical (non-classical) one because the diagonal coherent state distribution function is covariant under such transformations. Therefore, it is reasonable to require that any signature of non-classicality for a two-mode system, in particular SPS, be $U(2)$ invariant. To achieve this we regard all modes related to the original ones by passive $U(2)$ transformations as basically equivalent; then a survey of the SPS properties for each mode in this equivalence class of modes leads to the formulation of a $U(2)$ invariant definition of SPS. We search over the set of all modes for that one which minimizes the relevant parameter-measuring number fluctuation minus the mean. In this way, we arrive at that $U(2)$ combination of the two modes which is most likely to be manifestly subPoissonian. A much wider class of non-classical states can be explored using this formalism compared to the earlier ways of handling two-mode situations.

The material in this paper is arranged as follows. In section 2 we recapitulate the basic kinematics of two-mode systems and the action of the group $S p(4, \Re)$ on the nonHermitian annihilation and creation operators. The Hermitian generators of this action, and the maximal compact subgroup $U(2) \in S p(4, \Re)$, are recorded. The notion of $U(2)$ invariant SPS is then developed by regarding all modes related to one another by (passive) $S U$ (2) transformations as equivalent, and by minimizing the variable one-mode $Q$ parameter over the set of all (normalized) modes. The algebraic machinery needed to carry this out, for an arbitrary given state of the two-mode system, is set up. In section 3 we consider three applications: squeezed coherent states, squeezed thermal states, and a general superposition of two coherent states. In each case the analytic work is carried out as far as possible, and then we resort to numerical studies which are graphically displayed. Section 4 contains concluding remarks.

\section{2. $U(2)$ invariant definition of sub-Poissonian photon statistics for two-mode systems}

We consider two orthogonal modes of the radiation field, their orthogonality being achievable by their having different frequencies, orthogonal polarizations or different directions of propagation. These modes can be quantum mechanically described by photon annihilation operators $a_{r}$ and corresponding photon creation operators $a_{r}^{\dagger}$, where $r=1,2$. These operators can be arranged as a column vector $\xi^{(c)}$ :

$$
\xi^{(c)}=\left(\xi_{s}^{(c)}\right)=\left(\begin{array}{c}
a_{1} \\
a_{2} \\
a_{1}^{\dagger} \\
a_{2}^{\dagger}
\end{array}\right) \quad s=1, \ldots, 4 .
$$

The superscript $(c)$ on $\xi$ indicates that the entries here are complex, i.e. non-Hermitian. The quadrature components of these operators, which are the Hermitian phase-space variables $q$ 's and $p$ 's, can be written as another column vector, related to $\xi^{(c)}$ by a fixed numerical 
matrix $\Omega$

$$
\xi=\left(\xi_{s}\right)=\left(\begin{array}{c}
q_{1} \\
q_{2} \\
p_{1} \\
p_{2}
\end{array}\right)=\Omega^{-1} \xi^{(c)} \quad \Omega=\left(\Omega^{-1}\right)^{\dagger}=\frac{1}{\sqrt{2}}\left(\begin{array}{cccc}
1 & 0 & \mathrm{i} & 0 \\
0 & 1 & 0 & \mathrm{i} \\
1 & 0 & -\mathrm{i} & 0 \\
0 & 1 & 0 & -\mathrm{i}
\end{array}\right) .
$$

The canonical commutation relations obeyed by the creation and annihilation operators can be written in terms of $\xi$ or $\xi^{(c)}$ :

$$
\begin{aligned}
& {\left[\xi_{s}, \xi_{t}\right]=\mathrm{i} \beta_{s t}} \\
& {\left[\xi_{s}^{(c)}, \xi_{t}^{(c)}\right]=\beta_{s t}} \\
& \left(\beta_{s t}\right)=\left(\begin{array}{cccc}
0 & 0 & 1 & 0 \\
0 & 0 & 0 & 1 \\
-1 & 0 & 0 & 0 \\
0 & -1 & 0 & 0
\end{array}\right) .
\end{aligned}
$$

A general real linear homogeneous transformation on the $q$ 's and $p$ 's which preserves these commutation relations is described by a $4 \times 4$ real matrix $S$ obeying the condition

$$
S \beta S^{T}=\beta .
$$

This is the defining property for the elements of the non-compact group $\operatorname{Sp}(4, \Re)$

$$
S p(4, \Re)=\left\{S=4 \times 4 \text { real matrix } \mid S \beta S^{T}=\beta\right\} .
$$

When $\xi$ undergoes a transformation by $S \in S p(4, \Re)$, the non-Hermitian operators $\xi^{(c)}$ transform through a complex matrix $S^{(c)}$, obtained from $S$ by conjugation with $\Omega$ :

$$
\begin{array}{ll}
S \in S p(4, \Re) \quad & \xi^{\prime}=S \xi \Rightarrow \\
& \xi^{(c) \prime}=S^{(c)} \xi^{(c)} \\
& S^{(c)}=\Omega S \Omega^{\dagger} .
\end{array}
$$

The complex matrices $S^{(c)}$ are a faithful representation of the real matrix group $\operatorname{Sp}(4, \mathfrak{R})$. In this sense we will treat them as elements of $S p(4, \Re)$.

The maximal compact subgroup $U(2)$ of $\operatorname{Sp}(4, \Re)$ can be identified as follows:

$$
\begin{aligned}
& \mathcal{K} \equiv U(2)=\left\{S^{(c)}(U) \in \operatorname{Sp}(4, \Re) \mid U \in U(2)\right\} \subset \operatorname{Sp}(4, \Re) \\
& S^{(c)}(U)=\left(\begin{array}{cc}
U & 0 \\
0 & U^{\star}
\end{array}\right) .
\end{aligned}
$$

The block diagonal form is responsible for the fact that such transformations do not mix $a$ and $a^{\dagger}$; in fact $\mathcal{K}$ is the largest sub-group with this property.

Let $\mathcal{H}$ be the Hilbert space on which $\xi$ and $\xi^{(c)}$ act irreducibly. It follows from the Stone-von Neumann theorem [10] that, since the canonical commutation and Hermiticity relations are invariant under the transformation (2.6) for any $S^{(c)} \in S p(4, \Re)$, it is possible to construct a unitary operator $\mathcal{U}\left(S^{(c)}\right)$ on $\mathcal{H}$ implementing (2.6) via conjugation:

$$
\begin{aligned}
& S^{(c)} \in S p(4, \Re): S_{s t}^{(c)} \xi_{t}^{(c)}=\mathcal{U}\left(S^{(c)}\right)^{-1} \xi_{s}^{(c)} \mathcal{U}\left(S^{(c)}\right) \\
& \mathcal{U}\left(S^{(c)}\right)^{\dagger} \mathcal{U}\left(S^{(c)}\right)=\mathbf{1} \quad \text { on } \mathcal{H} .
\end{aligned}
$$

The generators of the operators $\mathcal{U}\left(S^{(c)}\right)$ are given by 10 independent, Hermitian, quadratic expressions in $a_{r}$ and $a_{r}^{\dagger}$. We define the four photon number-conserving generators $J_{0}, J_{j}$ 
and the six photon number non-conserving generators $K_{j}, L_{j}, j=1,2,3$ :

$$
\begin{aligned}
& J_{0}=\frac{1}{2}(N+1)=\frac{1}{2}\left(a_{1}^{\dagger} a_{1}+a_{2}^{\dagger} a_{2}+1\right) \\
& J_{1}=\frac{1}{2}\left(a_{1}^{\dagger} a_{2}+a_{2}^{\dagger} a_{1}\right) \\
& J_{2}=\frac{1}{2} \mathrm{i}\left(a_{2}^{\dagger} a_{1}-a_{1}^{\dagger} a_{2}\right) \\
& J_{3}=\frac{1}{2}\left(a_{1}^{\dagger} a_{1}-a_{2}^{\dagger} a_{2}\right) \\
& K_{1}=\frac{1}{4}\left(a_{1}^{\dagger 2}+a_{1}^{2}-a_{2}^{\dagger 2}-a_{2}^{2}\right) \\
& K_{2}=-\frac{1}{4} \mathrm{i}\left(a_{1}^{\dagger 2}-a_{1}^{2}+a_{2}^{\dagger 2}-a_{2}^{2}\right) \\
& K_{3}=-\frac{1}{2}\left(a_{1}^{\dagger} a_{2}^{\dagger}+a_{1} a_{2}\right) \\
& L_{1}=\frac{1}{4} \mathrm{i}\left(a_{1}^{\dagger 2}-a_{1}^{2}-a_{2}^{\dagger 2}+a_{2}^{2}\right) \\
& L_{2}=\frac{1}{4}\left(a_{1}^{\dagger 2}+a_{1}^{2}+a_{2}^{\dagger 2}+a_{2}^{2}\right) \\
& L_{3}=-\frac{1}{2} \mathrm{i}\left(a_{1}^{\dagger} a_{2}^{\dagger}-a_{1} a_{2}\right) .
\end{aligned}
$$

These generators obey the commutation relations

$$
\left[J_{j}, J_{k}\right]=\mathrm{i} \epsilon_{j k l} J_{l} \quad\left[J_{0}, J_{j}\right]=0
$$

$\left[J_{j}, K_{k}\right.$ or $\left.L_{k}\right]=\mathrm{i} \epsilon_{j k l}\left(K_{l}\right.$ or $\left.L_{l}\right) \quad\left[J_{0}, K_{j} \pm \mathrm{i} L_{j}\right]=\mp\left(K_{j} \pm \mathrm{i} L_{j}\right)$

$\left[K_{j}, K_{k}\right]=\left[L_{j}, L_{k}\right]=-\mathrm{i} \epsilon_{j k l} J_{l} \quad\left[K_{j}, L_{k}\right]=\mathrm{i} \delta_{j k} J_{0}$.

From the above commutation relations, it is clear that $J_{0}$ and $J_{j}$ form the algebra of $U(2)$ and hence generate the unitary operators corresponding to the elements of the maximal compact subgroup $\mathcal{K}$ of $S p(4, \Re)$. On the other hand, $K_{j}$ and $L_{j}$ are the generators of the unitary operators corresponding to the non-compact elements of $\operatorname{Sp}(4, \Re)$ and they do not form a closed algebra. These non-compact elements are recognized in the context of quadrature squeezing to be the squeezing transformations and their complete classification has been given elsewhere [4].

We now consider the notion of SPS for the physical states of a two-mode system. For one-mode systems, such an analysis is based on Mandel's $Q$ parameter [6]

$$
Q=\frac{\left\langle a^{\dagger 2} a^{2}\right\rangle-\left\langle a^{\dagger} a\right\rangle^{2}}{\left\langle a^{\dagger} a\right\rangle}
$$

where $a$ and $a^{\dagger}$ are the annihilation and creation operators for the one-mode radiation field, the expectation values being taken for the state of interest. The $Q$ parameter distinguishes between physical states as having Poissonian, sub-Poissonian and super-Poissonian photon statistics, as $Q$ is $0,<0$ and $>0$ for the above cases respectively. In particular, the states with negative $Q$ are non-classical, in the sense that such a distribution can not be derived from any classical statistical ensemble. Therefore, in this limited sense, the $Q$ parameter can be used to classify states as classical and non-classical. More precisely, $Q<0(>0)$ is a sufficient (necessary) condition for non-classicality (classicality). We may note in passing that the denominator in $Q$ is chosen as a normalizing factor purely by convention, and in all the preceding statements only the sign of $Q$ has been relevant.

For a situation involving two modes, the notion of SPS defined above is not adequate, and needs to be suitably generalized. As it stands, one can analyse the photon statistics of one of the modes, or a preselected linear combination of both. Then again, for a given state, this mode which one chooses may not be the one in which the photon number distribution may be non-classical. Hence the sign of $Q$ for a preselected mode may not disclose the non-classical nature of the two-mode state, even if it is non-classical. This clearly indicates 
that an intrinsically two-mode notion of SPS, allowing for the possibility of forming all possible normalized superpositions of the two modes, is required.

The standard way [1] of distinguishing classical from non-classical states (already implicitly assumed in the above) is through the diagonal coherent state description. The general two-mode coherent state with complex two-component displacement $\tilde{z}=\left(z_{1}, z_{2}\right)$ is defined by

$$
\begin{aligned}
|\tilde{z}\rangle & =\exp \left(\tilde{z} \cdot \tilde{a}^{\dagger}-\tilde{z}^{\star} \cdot \tilde{a}\right)|0,0\rangle \\
& =\exp \left(-\frac{1}{2}\left|z_{1}\right|^{2}-\frac{1}{2}\left|z_{2}\right|^{2}\right) \exp \left(z_{1} a_{1}^{\dagger}+z_{2} a_{2}^{\dagger}\right)|0,0\rangle .
\end{aligned}
$$

These are normalized states and form an over-complete set. A given two-mode density operator $\rho$ can be expanded in terms of them

$$
\rho=\int \frac{\mathrm{d}^{2} z_{1} \mathrm{~d}^{2} z_{2}}{\pi^{2}} \phi\left(z_{1}, z_{2}\right)\left|z_{1}, z_{2}\right\rangle\left\langle z_{1}, z_{2}\right| .
$$

The unique normalized weight function $\phi\left(z_{1}, z_{2}\right)$ gives the complete description of the twomode state and can in general be a distribution which is quite singular [11]. In the case when $\phi\left(z_{1}, z_{2}\right)$ can be interpreted as a probability distribution (i.e. it is non-negative and is nowhere more singular than a delta function), equation (2.13) implies that the state $\rho$ is a classical mixture of coherent states which have a natural classical limit. Such quantum states are referred to as classical; in contrast the others, for which $\phi\left(z_{1}, z_{2}\right)$ either becomes negative or more singular than a delta function somewhere, are defined as being nonclassical. This classification is general and can be done for any number of modes. In particular, for the one-mode case, the states having negative $Q$ are a subset of the states with non-classical diagonal coherent-state distribution functions. Here we may emphasize that quadrature squeezing, SPS (and various other effects), are independent signatures of non-classicality - a given state may show one and not the other, or neither, and yet be non-classical, this showing up in some higher-order effects.

When the two-mode state, with density matrix $\rho$, transforms under a unitary operator corresponding to the compact $U(2)$ subgroup of $S p(4, \Re)$, the distribution $\phi\left(z_{1}, z_{2}\right)$ undergoes a point transformation given in terms of the $U(2)$ matrix $U \in U(2)$ :

$$
\begin{aligned}
& \rho^{\prime}=\mathcal{U}\left(S^{(c)}(U)\right) \rho \mathcal{U}\left(S^{(c)}(U)\right)^{-1} \Leftrightarrow \phi^{\prime}\left(z_{1}, z_{2}\right)=\phi\left(z_{1}^{\prime}, z_{2}^{\prime}\right) \\
& \left(\begin{array}{l}
z_{1}^{\prime} \\
z_{2}^{\prime}
\end{array}\right)=U\left(\begin{array}{l}
z_{1} \\
z_{2}
\end{array}\right) .
\end{aligned}
$$

Thus, under $U(2)$ transformations classical states map on to classical ones and non-classical states to non-classical ones; these transformations are incapable of generating a non-classical state from a classical one. Therefore, it is reasonable to demand that any signature of nonclassicality be invariant under such transformations.

At this stage, we recapitulate and collect some interesting and important properties of the maximal compact subgroup $\mathcal{K}$ of $\operatorname{Sp}(4, \Re)$.

(a) As is clear from equation (2.7), when $\xi^{(c)}$ undergoes a $U(2)$ transformation, the annihilation operators $a_{r}$ 's are not mixed with the creation operators $a_{r}^{\dagger}$ 's.

(b) The action of the elements of $U(2)$ (generated by $J_{0}$ and $J_{j}$ ) on a state does not change the total photon number or its distribution.

(c) The diagonal coherent state distribution function is covariant under $U(2)$ transformations.

(d) One requires only passive optical elements to experimentally implement any $U(2)$ transformation on a state of the two-mode electromagnetic field [12]. 
Motivated by the above considerations we now define an intrinsically two-mode and $U(2)$ invariant notion of SPS. For the purpose of our present analysis it is convenient to write the $U$ (2) transformed mode operators in terms of two column vectors $\mathcal{A}$ and $\alpha$ :

$$
\mathcal{A}=\left(\begin{array}{l}
a_{1} \\
a_{2}
\end{array}\right) \quad \alpha=\left(\begin{array}{c}
\alpha_{1} \\
\alpha_{2}
\end{array}\right)
$$

where $\alpha_{1}$ and $\alpha_{2}$ are complex numbers such that

$$
\begin{aligned}
& U(\alpha)=\left(\begin{array}{cc}
\alpha_{1}^{\star} & \alpha_{2}^{\star} \\
-\alpha_{2} & \alpha_{1}
\end{array}\right) \in S U(2) \quad\left|\alpha_{1}\right|^{2}+\left|\alpha_{2}\right|^{2}=1 \\
& U(\alpha, \psi)=\left(\begin{array}{cc}
\alpha_{1}^{\star} & \alpha_{2}^{\star} \\
-\mathrm{e}^{\mathrm{i} \psi} \alpha_{2} & \mathrm{e}^{\mathrm{i} \psi} \alpha_{1}
\end{array}\right) \in U(2) \quad 0 \leqslant \psi \leqslant 2 \pi .
\end{aligned}
$$

When $\xi^{(c)}$ undergoes a $U(2)$ transformation given by $U(\alpha, \psi)$, the annihilation and creation operators for the transformed first mode can be written in terms of $\mathcal{A}$ and $\alpha$ alone:

$$
\begin{aligned}
& a(\alpha)=\alpha^{\dagger} \mathcal{A}=\alpha_{1}^{\star} a_{1}+\alpha_{2}^{\star} a_{2} \\
& a(\alpha)^{\dagger}=\mathcal{A}^{\dagger} \alpha=\alpha_{1} a_{1}^{\dagger}+\alpha_{2} a_{2}^{\dagger} .
\end{aligned}
$$

Thus the most general normalized 'first mode' after the $U(2)$ transformation is determined by $S U(2) \in U(2)$ independent of $\psi$. This particular mode will henceforth be called the $S U$ (2) transformed mode, and $\alpha$ will be used to denote the $S U(2)$ element involved.

Let $\rho$ be the density matrix for any (pure or mixed) state of the two-mode radiation field. Then we can define the following function:

$$
\begin{aligned}
Q(\rho ; \alpha) & =\frac{\left\langle a(\alpha)^{\dagger 2} a(\alpha)^{2}\right\rangle_{\rho}-\left\langle a(\alpha)^{\dagger} a(\alpha)\right\rangle_{\rho}^{2}}{\left\langle\mathcal{A}^{\dagger} \mathcal{A}\right\rangle_{\rho}} \\
& =\frac{\operatorname{Tr}\left(\rho a(\alpha)^{\dagger 2} a(\alpha)^{2}\right)-\left(\operatorname{Tr}\left(\rho a(\alpha)^{\dagger} a(\alpha)\right)\right)^{2}}{\operatorname{Tr}\left(\rho \mathcal{A}^{\dagger} \mathcal{A}\right)}
\end{aligned}
$$

which is similar to the Mandel $Q$ parameter for the $S U(2)$ transformed mode $a(\alpha)$. Here we have chosen the normalizing denominator factor, which is essentially a matter of convention, to be $U(2)$ invariant, a natural requirement in the present context. When the state $\rho$ is transformed by the unitary operator $\mathcal{U}\left(S^{(c)}(U)\right)$ for some $U \in U(2)$, the function $Q(\rho ; \alpha)$ can be shown to change covariantly:

$$
\begin{aligned}
& S^{(c)}(U) \in \mathcal{K}: \rho^{\prime}=\mathcal{U}\left(S^{(c)}(U)\right) \rho \mathcal{U}\left(S^{(c)}(U)\right)^{-1} \Rightarrow \\
& Q\left(\rho^{\prime} ; \alpha\right)=Q\left(\rho ; \alpha^{\prime}\right) \quad \alpha^{\prime}=U \alpha .
\end{aligned}
$$

Now an overall phase change corresponding to elements in the $U(1)$ subgroup of $U(2)$ actually leaves $Q(\rho ; \alpha)$ unchanged, therefore no dependence on $\psi$ has been shown. So we have the freedom of running over all $\alpha$ 's $\in S U(2)$, i.e. we can choose various linear combinations of the two modes involved, related to each other by $S U(2)$ transformations. Since we want to look for the signature of the non-classical nature (if present) as manifested in the photon statistics, we vary $\alpha$ till we reach the minimum value of the function $Q(\rho ; \alpha)$ :

$$
Q(\rho)=\operatorname{Min}_{\substack{\text { over all } \\ \alpha \in S U(2)}} Q(\rho ; \alpha)=Q(\rho ; \bar{\alpha}) \quad \text { s.t. } Q(\rho ; \bar{\alpha}) \leqslant Q(\rho ; \alpha) .
$$

If $Q(\rho)<0$ we shall conclude that the photon number distribution for the two-mode state $\rho$ is non-classical and sub-Poissonian, or amplitude squeezed. This is our $U(2)$ invariant definition of SPS for states of two-mode fields. The mode in which the sub-Poissonian nature is manifest to the maximum degree is $a(\bar{\alpha})$. 
We shall refer to the expression $Q(\rho ; \alpha)$ and its minimum $Q(\rho)$ as the covariant and the invariant Mandel parameters respectively, for the two-mode state $\rho$. We now develop suitable formulae to handle the $\rho$ and $\alpha$ dependence of $Q(\rho ; \alpha)$. The numerator in our definition of $Q(\rho ; \alpha)$ consists of two terms, one arising from the expectation values of quadratic expressions in the creation and annihilation operators and the other arising from the expectation values of quartic terms. The quadratic term can be written:

$$
\begin{aligned}
& \operatorname{Tr}\left(\rho a(\alpha)^{\dagger} a(\alpha)\right)=s+\tilde{u} \cdot \tilde{q} \\
& \tilde{q}=\tilde{q}(\alpha)=\alpha^{\dagger} \tilde{\sigma} \alpha
\end{aligned}
$$

with the dependence on the state $\rho$ and on $\alpha \in S U(2)$ being clearly separated. The statedependent variables $s$ and $\tilde{u}$ transform under $S U(2)$ like a scalar and a Cartesian vector respectively, and can be evaluated from the equation

$$
\operatorname{Tr}\left(\rho a_{s}^{\dagger} a_{r}\right)=s \delta_{r s}+u_{j}\left(\sigma_{j}\right)_{r s} \quad r, s=1,2
$$

where $\sigma$ are the Pauli matrices. The term involving the expectation values of quartics in $a_{r}$ and $a_{r}^{\dagger}$ can be written in terms of the non-compact generators $\tilde{K}$ and $\tilde{L}$ of $\operatorname{Sp}(4, \Re)$, and a vector $\tilde{\lambda}$ representing the $S U(2)$ element involved:

$$
\begin{aligned}
& \operatorname{Tr}\left(\rho a(\alpha)^{\dagger^{2}} a(\alpha)^{2}\right)=\frac{1}{4} \lambda_{j} \lambda_{k}^{\star} H_{j k} \\
& H_{j k}=H_{k j}^{\star}=\operatorname{Tr}\left(\rho\left(K_{j}-\mathrm{i} L_{j}\right)\left(K_{k}+\mathrm{i} L_{k}\right)\right) \quad j, k=1,2,3 \\
& \tilde{\lambda}=\tilde{\lambda}(\alpha)=-\mathrm{i} \alpha^{T} \sigma_{2} \tilde{\sigma} \alpha \quad \tilde{\lambda}(\alpha) \tilde{\lambda}(\alpha)=0 .
\end{aligned}
$$

The Hermitian matrix $H$ can be written in terms of two real matrices, the real symmetric $R$ and real antisymmetric $S$, as $H=R+\mathrm{i} S$. The matrix $R$ transforms under $S U(2)$ as a second rank tensor whereas the matrix $S$ can be represented by a Cartesian vector $\tilde{v}$ under $S U$ (2), related to $S$ by $v_{j}=\frac{1}{2} \epsilon_{j k l} S_{k l}$.

The denominator of $Q(\rho ; \alpha)$ is $U(2)$ invariant since the operator $\mathcal{A}^{\dagger} \mathcal{A}=a_{1}^{\dagger} a_{1}+a_{2}^{\dagger} a_{2}$ is $U(2)$ invariant; it does not depend upon $\alpha$ and can be written in terms of $s$ as

$$
\mathcal{D}(Q(\rho ; \alpha))=\operatorname{Tr}\left(\rho \mathcal{A}^{\dagger} \mathcal{A}\right)=2 s .
$$

After some algebra, the complex vector $\tilde{\lambda}$ can be eliminated in favour of the real vector $\tilde{q}$, and $Q(\rho ; \alpha)$ can be written in terms of the state-dependent symmetric second-rank tensor $R$, the vectors $\tilde{u}, \tilde{v}$ and the scalar $s$ as

$$
Q(\rho ; \alpha)=Q(\rho ; \tilde{q}(\alpha))=\frac{1}{8 s}\left(\operatorname{Tr} R-q_{j} q_{k} R_{j k}+2 \tilde{v} \cdot \tilde{q}-4(s+\tilde{u} \cdot \tilde{q})^{2}\right) .
$$

Using the $U(2)$ covariance of $Q(\rho ; \alpha)$, we can assume without loss of generality that the real symmetric matrix $R$ is diagonal, and equation (2.25) then takes the simpler form

$$
Q(\rho ; \tilde{q}(\alpha))=\frac{1}{8 s}\left(\operatorname{Tr} R-\sum_{j} q_{j}^{2} R_{j j}+2 \tilde{v} \cdot \tilde{q}-4(s+\tilde{u} \cdot \tilde{q})^{2}\right) .
$$

The dependence of $Q(\rho ; \tilde{q}(\alpha))$ on $\alpha \in S U(2)$ is through the real unit vector $\tilde{q}(\alpha)$, which can be represented on the surface of a unit sphere. In order to obtain the invariant Mandel parameter $Q(\rho)$ for a given two-mode state, we have to minimize $Q(\rho ; \tilde{q}(\alpha))$ with respect to $\tilde{q}(\alpha)$, the parameters $R, \tilde{v}, s, \tilde{u}$ being determined by $\rho$. The most convenient coordinates which one chooses on the surface of the sphere to carry out this minimization will depend upon the physical state $\rho$ under consideration. 


\section{Application to two-mode squeezed coherent states, squeezed thermal states and superposition of coherent states}

In this section, we apply the formalism developed in section 2 to various interesting twomode states. Here we will see the relationship with the classification of two-mode squeezing transformations given in [4].

\subsection{The case of squeezed coherent states}

The most general (quadrature) squeezed coherent state is obtained by applying the operator $\mathcal{U}(\tilde{k}, \tilde{l})=\mathrm{e}^{\mathrm{i}(\tilde{k} \cdot \tilde{K}+\tilde{l} \cdot \tilde{L})}$ to the two-mode coherent state $\left|z_{1}, z_{2}\right\rangle$ defined in equation (2.12), for some complex $z_{1}, z_{2}$, where $\tilde{K}$ and $\tilde{L}$ are the non-compact generators of $\operatorname{Sp}(4, \Re)$ defined in equation (2.9) and $\tilde{k}$ and $\tilde{l}$ are real vectors. The operator $\mathcal{U}(\tilde{k}, \tilde{l})$ is conjugate to $\mathcal{U}^{(0)}(a, b)=\operatorname{expi}\left(a K_{2}+b L_{1}\right)$ for some $a \geqslant b \geqslant 0$, via an operator $\mathcal{U}\left(S^{c}(U)\right)$ :

$$
\begin{aligned}
& \mathcal{U}(\tilde{k}, \tilde{l})=\mathcal{U}^{-1}\left(S^{(c)}(U)\right) \mathcal{U}^{(0)}(a, b) \mathcal{U}\left(S^{(c)}(U)\right) \\
& \mathcal{U}^{(0)}(a, b)=\exp \left(\frac{(a-b)}{4}\left(a_{1}^{\dagger^{2}}-a_{1}^{2}\right)\right) \exp \left(\frac{(a+b)}{4}\left(a_{2}^{\dagger^{2}}-a_{2}^{2}\right)\right) .
\end{aligned}
$$

Each $\mathcal{U}^{(0)}(a, b)$ is a representative of an equivalence class of two-mode squeezing transformations. For $a=b$ we have the essentially single mode case, while for $b=0$ we have maximal involvement of the two modes. For the minimization of the $U(2)$ covariant $Q(\rho ; \alpha)$, the overall $U(2)$ factor $\mathcal{U}^{-1}\left(S^{(c)}(U)\right)$ is irrelevant. Also, the action of the operator $\mathcal{U}\left(S^{(c)}(U)\right)$ on $\left|z_{1}, z_{2}\right\rangle$ transforms it into another coherent state $\left|z_{1}^{\prime}, z_{2}^{\prime}\right\rangle$, with $z_{1}^{\prime}, z_{2}^{\prime}$ related to $z_{1}, z_{2}$ through the corresponding $U(2)$ transformation. Thus it suffices to examine the particular class of squeezed coherent states

$$
\left|z_{1}, z_{2}, a, b\right\rangle=\mathcal{U}^{(0)}(a, b)\left|z_{1}, z_{2}\right\rangle .
$$

A complete discussion of the two-mode squeezing transformations and squeezed states has been given in [4].

The covariant Mandel parameter $Q\left(z_{1}, z_{2}, a, b ; \tilde{q}(\alpha)\right)$ for the $S U(2)$ transformed mode for squeezed coherent states can be calculated by straightforward algebra and turns out to be rather lengthy. The complete expression is given in the appendix (equation (A.1)), not so much to burden the reader as to show the result in the physically important case of squeezed coherent states. $Q\left(z_{1}, z_{2}, a, b ; \tilde{q}(\alpha)\right)$ depends on $a, b$ through hyperbolic functions and on $\left|z_{1}\right|,\left|z_{2}\right|$ through polynomial functions. Its dependence on the phases of $z_{1}$ and $z_{2}$ and the polar coordinates on the surface of the unit sphere describing the unit vector $\tilde{q}(\alpha)$, is through trigonometric functions and is oscillatory in nature. In order to obtain the invariant Mandel parameter $Q(\rho)$, this function has to be minimized with respect to $\tilde{q}(\alpha)$. Since this is not possible analytically, the results obtained numerically are displayed in figures 1,2 and $3 \dagger$.

In each figure, we plot the minimum value of $Q\left(z_{1}, z_{2}, a, b ; \tilde{q}(\alpha)\right)$ as a function of the squeeze factors $a$ and $b$, keeping the complex displacements $z_{1}$ and $z_{2}$ fixed. Figure 1(a) displays the results for the squeezed vacuum; this never shows SPS. Incidentally this case already illustrates the independence of different signatures of non-classicality, since quadrature squeezing is present but not SPS. The plots of figures $1(b)-(d)$ on the other hand are obtained by varying the phase of one of the displacements $\left(z_{2}\right)$, keeping its magnitude fixed, with the other displacement $\left(z_{1}\right)$ being zero. Different values for the phase of the non-zero displacement give qualitatively different results; in particular when this phase is

$\dagger$ Note that the subfigures in each figure are not drawn to the same scale. 

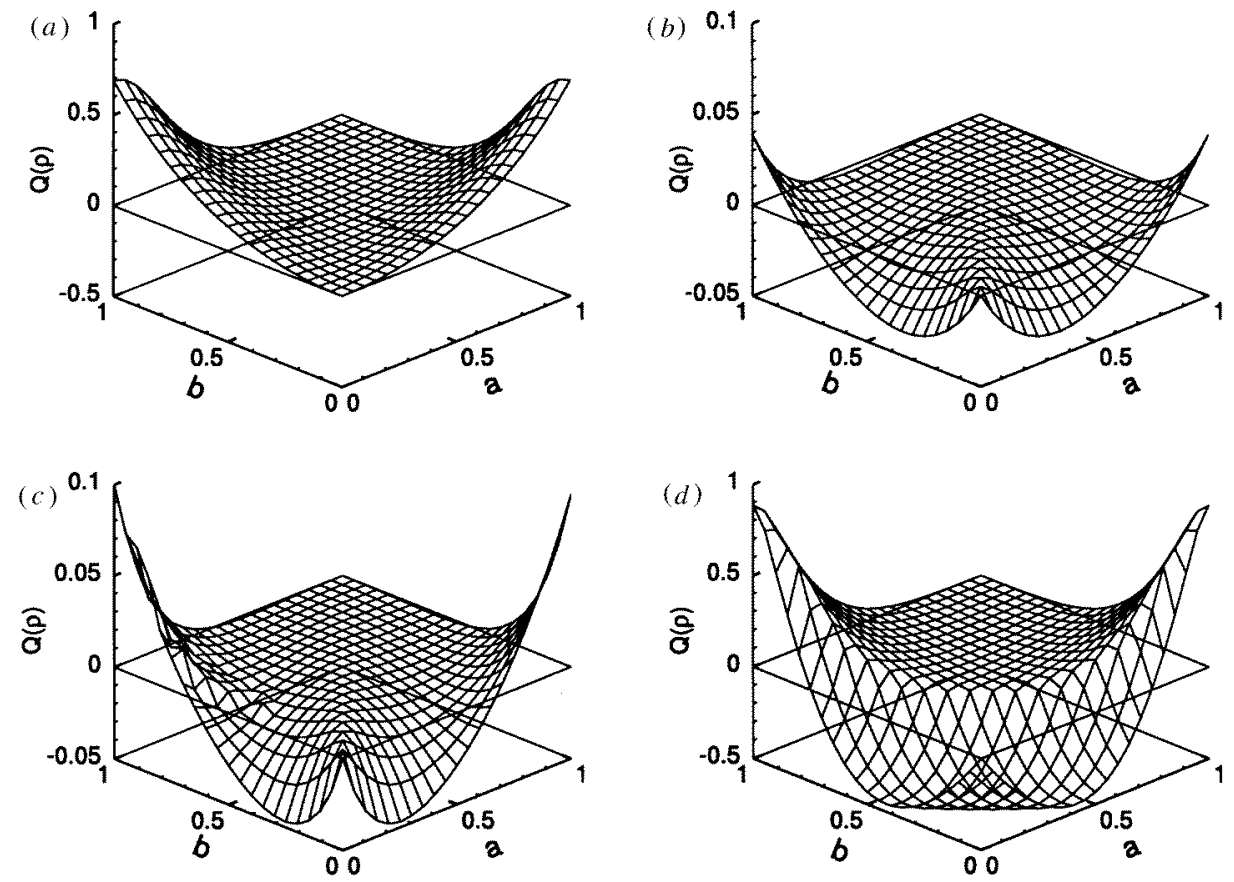

Figure 1. Plots of the invariant Mandel parameter $Q(\rho)$ for squeezed coherent states as a function of squeeze parameters $a$ and $b$. (a) The plot for squeezed vacuum, i.e. $z_{1}=z_{2}=0$. (b)-(d) The plots for $\left|z_{1}\right|=0,\left|z_{2}\right|=3.0$ and the phase of $z_{2}$ taking the values $0, \pi / 4$ and $\pi / 2$, respectively.

$\frac{\pi}{2}$, as is clear from figure $1(d)$ even some of the essentially single-mode states lying along $a=b$ show SPS. In figure 2 we choose equal magnitudes of displacements for the two modes; plots have been generated for different values of their phases. The displacement parameters in figure 3 are unequal in magnitude; four plots have been given for the same choices of phase values as in the corresponding plots in figure 2. The qualitative features of individual plots are similar to the corresponding plots in figure 2 though the actual values of the invariant Mandel parameter are different.

We now make some general remarks about the results described above. In all the plots of figures 1,2 and 3, every point in the region $b>a$ can be mapped onto a corresponding unique point in some region $a>b$ (which in general is not in the same figure), through that $U(2)$ transformation of the displacements $z_{1}$ and $z_{2}$, which effectively changes $\mathcal{U}^{(0)}(a, b)$ to $\mathcal{U}^{(0)}(b, a)$. Whenever the displacement parameters are invariant under this particular $U(2)$ transformation, the plot has a symmetry about the line $a=b$; as in all the plots of figure 1 . Such a symmetry is not exhibited by the plots of figures 2 and 3 . In all the plots the invariant Mandel parameter is zero or negative along the line $a=b$, i.e. for the subset of essentially single-mode squeezed states. This happens because, even though the choice of displacement parameters is such that the single mode which is squeezed has super-Poissonian statistics $(Q>0)$, the minimization chooses the other mode which is in a coherent state $(Q=0)$. Apart from the case of a squeezed vacuum (figure 1 $(a)$ ) all other choices of displacement show SPS for some values of the squeeze parameters $a, b$. When squeezing becomes large in comparison to the displacement, and we are away from the line $a=b$, SPS disappears and the states tend to become more and more super-Poissonian. 

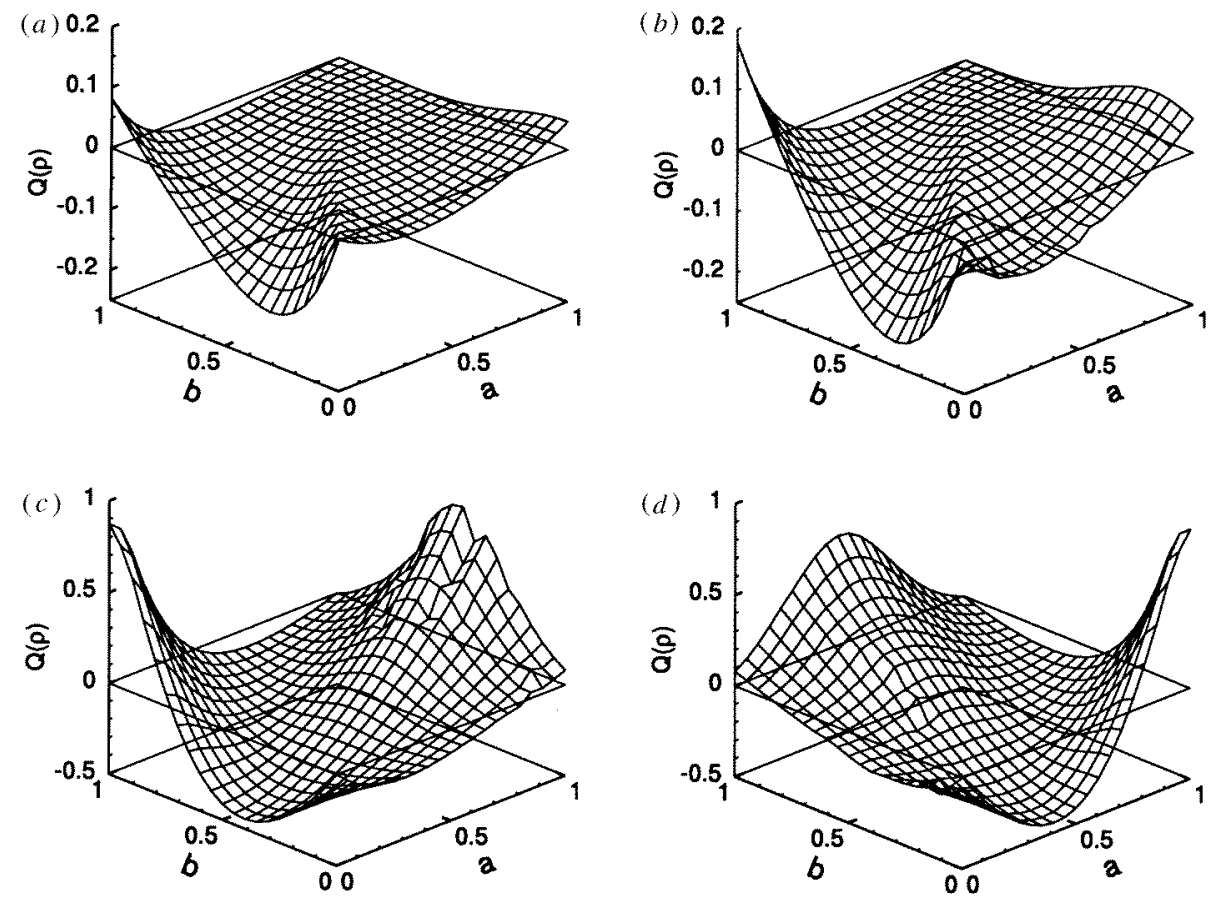

Figure 2. Plots of the invariant Mandel parameter $Q(\rho)$ for squeezed coherent states as a function of squeeze parameters $a$ and $b$ for the case when the magnitudes of the displacements in the two modes are equal: $\left|z_{1}\right|=\left|z_{2}\right|=2.0$. The values of the phases of $z_{1}$ and $z_{2}$ in $(a)-(d)$ are $(0,0),(0, \pi / 4),(0, \pi / 2)$ and $(\pi / 2, \pi / 2)$, respectively.

\subsection{The case of squeezed thermal states}

We next look at the case of a two-mode isotropic thermal state subjected to squeezing. The normalized density operator corresponding to the inverse temperature $\beta=\hbar \omega / k T$ is explicitly $U(2)$ invariant and described in the Fock representation by

$$
\begin{aligned}
\rho_{0}(\beta) & =\left(1-\mathrm{e}^{-\beta}\right)^{2} \exp \left[-\beta\left(a_{1}^{\dagger} a_{1}+a_{2}^{\dagger} a_{2}\right)\right] \\
& =\left(1-\mathrm{e}^{-\beta}\right)^{2} \sum_{n_{1}, n_{2}=0}^{\infty} \mathrm{e}^{-\beta\left(n_{1}+n_{2}\right)}\left|n_{1}, n_{2}\right\rangle\left\langle n_{1}, n_{2}\right|
\end{aligned}
$$

with $U(2)$ invariance expressed by

$$
\mathrm{e}^{\mathrm{i} \theta J_{0}} \rho_{0}(\beta) \mathrm{e}^{-i \theta J_{0}}=\mathrm{e}^{\mathrm{i} \alpha \cdot J} \rho_{0}(\beta) \mathrm{e}^{-\mathrm{i} \alpha \cdot J}=\rho_{0}(\beta) .
$$

Therefore it suffices to examine the properties of the density operator obtained by conjugating $\rho_{0}(\beta)$ with $\mathcal{U}^{(0)}(a, b)$

$$
\rho(\beta ; a, b)=\mathcal{U}^{(0)}(a, b) \rho_{0}(\beta) \mathcal{U}^{(0)}(a, b)^{-1} .
$$

In contrast to the previous case, now the covariant Mandel parameter $Q(\beta ; a, b ; \tilde{q}(\alpha))$ for the state $\rho(\beta ; a, b)$ is calculable by straightforward algebra:

$$
\begin{gathered}
Q(\beta ; a, b ; \tilde{q}(\alpha))=\left[\left(\mathrm{e}^{\beta}-1\right)\left(2\left(1-\mathrm{e}^{\beta}\right)+2\left(1+\mathrm{e}^{\beta}\right) \cosh (2 a) \cosh (2 b)\right)\right]^{-1} \\
\times\left[\frac { 1 } { 4 } \left(( 1 - q _ { 3 } ^ { 2 } ) \left(2\left(1-\mathrm{e}^{\beta}\right)^{2}+4\left(1-\mathrm{e}^{2 \beta}\right) \cosh (2 a) \cosh (2 b)\right.\right.\right. \\
\left.+\left(1+\mathrm{e}^{\beta}\right)^{2}(\cosh (4 a)+\cosh (4 b))\right)
\end{gathered}
$$



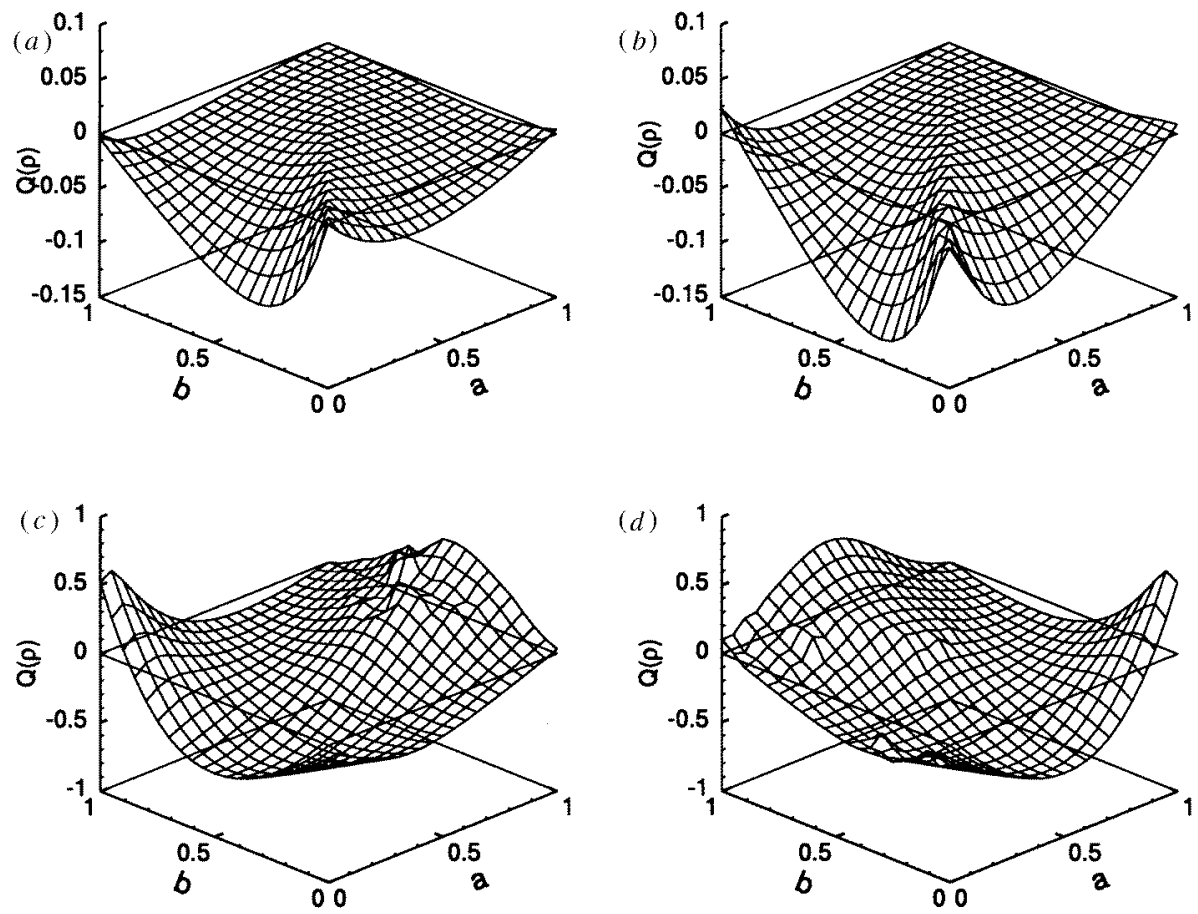

Figure 3. Plots of the invariant Mandel parameter $Q(\rho)$ for squeezed coherent states as a function of squeeze parameters $a$ and $b$ for the case when the magnitudes of the displacements in the two modes are unequal: $\left|z_{1}\right|=2.0$ and $\left|z_{2}\right|=4.0$. The values of the phases of $z_{1}$ and $z_{2}$ in $(a)-(d)$ are $(0,0),(0, \pi / 4),(0, \pi / 2)$ and $(\pi / 2, \pi / 2)$, respectively.

$$
\begin{aligned}
& +\left(1+\mathrm{e}^{\beta}\right)^{2}\left(q_{1}^{2}-q_{2}^{2}\right)(\cosh (4 a)-\cosh (4 b)) \\
& -\frac{1}{2}\left(1+q_{3}^{2}\right)\left(10-12 \mathrm{e}^{\beta}+10 \mathrm{e}^{2 \beta}+16\left(1-\mathrm{e}^{2 \beta}\right) \cosh (2 a) \cosh (2 b)\right. \\
& \left.\left.+6\left(1+\mathrm{e}^{\beta}\right)^{2} \cosh (4 a) \cosh (4 b)\right)\right) \\
& -\frac{1}{2}\left(\left(1+\mathrm{e}^{\beta}\right) q_{3}\left(4-4 \mathrm{e}^{\beta}+6\left(1+\mathrm{e}^{\beta}\right) \cosh (2 a) \cosh (2 b)\right) \sinh (2 a) \sinh (2 b)\right) \\
& -\frac{1}{2}\left(\left(2-2 \mathrm{e}^{\beta}+2\left(-1+\mathrm{e}^{2 \beta}\right) \cosh (2 a) \cosh (2 b)\right)\right. \\
& \left.\left.+\left(\left(1+\mathrm{e}^{\beta}\right) q_{3} \sinh (2 a) \sinh (2 b)\right)\right)^{2}\right]
\end{aligned}
$$

here $q_{1}, q_{2}, q_{3}$ are the Cartesian components of $\tilde{q}$ with $q_{1}^{2}+q_{2}^{2}+q_{3}^{2}=1$.

The minimum value of the function $Q(\beta, a, b, \tilde{q}(\alpha))$, the parameter $Q(\rho(\beta, a, b))$, can be calculated analytically. The state $\rho(\beta, a, b)$ being the squeezed thermal state is always super-Poissonian. For a given temperature (given $\beta$ ) this super-Poissonian nature is least for the case when only one mode is squeezed $(a=b)$, increases as the squeezing becomes increasingly two mode in nature, and finally is maximum when the state is maximally twomode squeezed, i.e. when $a=0(b=0)$ for a given $b(a)$. When the temperature is changed the states with higher temperatures (lower $\beta$ ) are more super-Poissonian compared to the ones at lower temperatures (higher $\beta$ ). Thus for fixed $a$ and $b, Q(\beta ; a, b)$ increases as $\beta$ decreases. The actual plots of $Q(\rho(\beta, a, b))$ as a function of $a, b$ are given at different temperatures in figure $4 \dagger$.

$\dagger$ Note that the subfigures in each figure are not drawn to the same scale. 

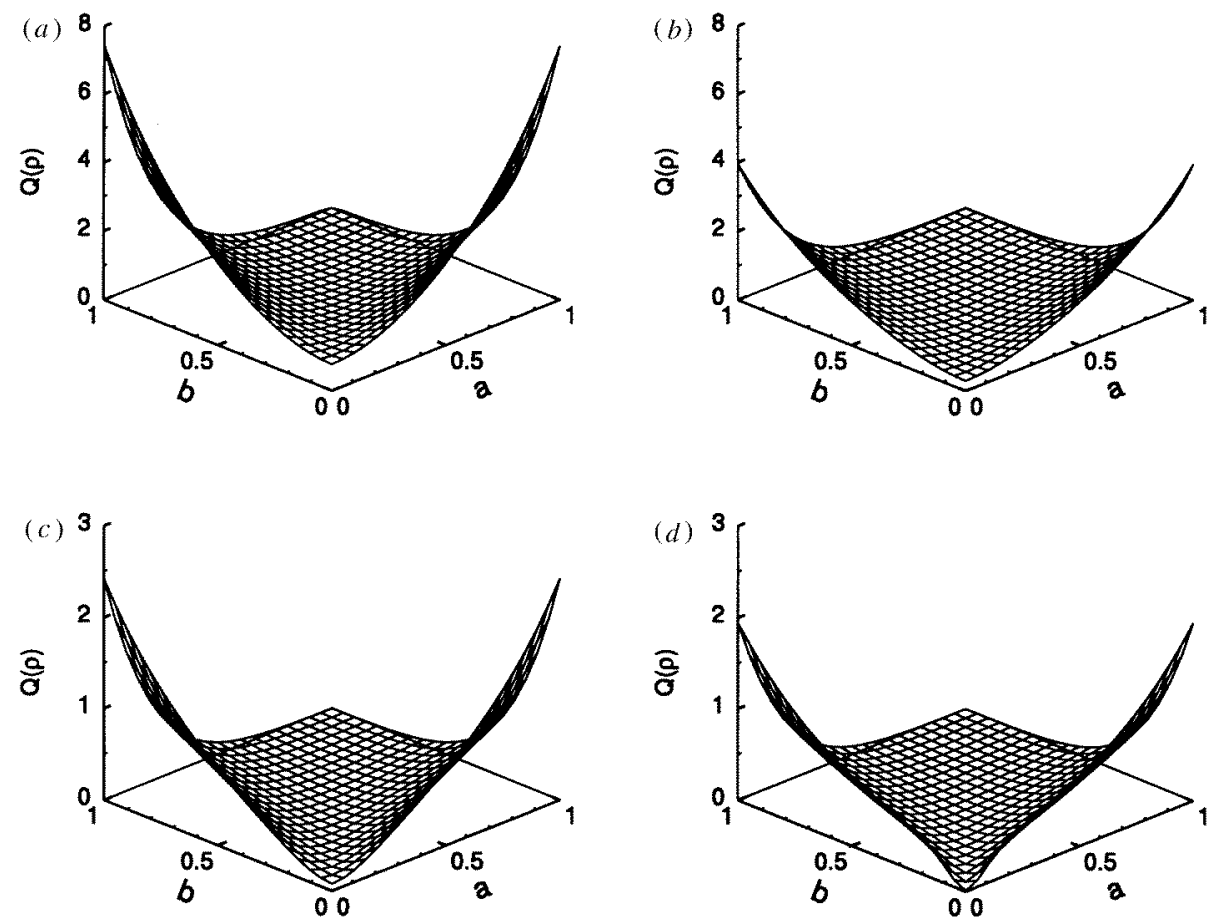

Figure 4. Plots of the invariant Mandel parameter $Q(\rho)$ for squeezed thermal states as a function of squeeze parameters $a$ and $b$ at different inverse temperatures; $\beta$ takes the values $0.5,1.0,2.0$ and 4.0 in $(a)-(d)$, respectively.

It is interesting to note that the particular mode for which the function $Q(\rho(\beta, a, b), \tilde{q}(\alpha))$ is minimum turns out to be one of the original modes, corresponding to $q_{3}= \pm 1$. This happens because the thermal state density matrix $\rho_{0}(\beta)$ is explicitly $U(2)$ invariant and the representative two-mode squeezing operator $\mathcal{U}^{(0)}(a, b)$ can be factorized into two commuting operators, each pertaining to one of the original modes see equation (3.1). In general, for a different choice of the representative operators, the minima could occur at an arbitrary $S U(2)$ transformed first mode. All the plots of figure 4 are symmetric about the line $a=b$ because of the explicit $U(2)$ invariance of the thermal-state density matrix $\rho_{0}(\beta)$ (equation (3.4)).

\subsection{The case of superposition of coherent states}

Lastly we apply our formalism to the superposition of two two-mode coherent states. In this case, no squeezing transformation $\mathcal{U}^{(0)}(a, b)$ is involved. For simplicity we consider only the case with real displacements.

A general superposition of two two-mode coherent states with real displacements and a phase difference $\eta$ between them is given by

$$
\left|\psi\left(u_{1}, u_{2}, v_{1}, v_{2}, r, \eta\right)\right\rangle=\frac{1}{N}\left(\left|u_{1}, u_{2}\right\rangle+r \exp (\mathrm{i} \eta)\left|v_{1}, v_{2}\right\rangle\right)
$$

where

$$
N^{2}=1+r^{2}+2 r \cos \eta \exp \left(-\frac{1}{2}\left(u_{1}^{2}+u_{2}^{2}+v_{1}^{2}+v_{2}^{2}\right)+u_{1} v_{1}+u_{2} v_{2}\right) .
$$


With the help of a $U(2)$ transformation, without any loss of generality we can set $v_{2}=0$ and thus it suffices to study only the states $\left|\psi\left(u_{1}, u_{2}, v_{1}, 0, r, \eta\right)\right\rangle$. The covariant Mandel parameter $Q\left(u_{1}, u_{2}, v_{1}, r, \eta ; \tilde{q}(\alpha)\right)$ for this superposition of coherent states is given in terms of the polar coordinates $\theta$ and $\phi$ on the surface of the sphere representing $\tilde{q}$ as

$$
\begin{aligned}
Q\left(u_{1}, u_{2}, v_{1}, r\right. & , \eta \tilde{q}(\alpha))=\left[4\left(u_{1}^{2}+u_{2}^{2}+r^{2} v_{1}^{2}+2 \mathrm{e}^{-\frac{1}{2}\left(u_{1}^{2}+u_{2}^{2}+v_{1}^{2}\right)+u_{1} v_{1}} r u_{1} v_{1} \cos (\eta)\right)\right]^{-1} \\
& \times\left[\left(1+r^{2}+2 \mathrm{e}^{-\frac{1}{2}\left(u_{2}^{2}+\left(u_{1}-v_{1}\right)^{2}\right)} r \cos (\eta)\right)\right. \\
& \times\left(4\left(u_{1}^{4}+r^{2} v_{1}^{4}\right) \cos \left(\frac{\theta}{2}\right)^{4}+4 u_{2}^{4} \sin \left(\frac{\theta}{2}\right)^{4}+8 u_{1}^{3} u_{2} \cos \left(\frac{\theta}{2}\right)^{2} \cos (\phi) \sin (\theta)\right. \\
& \left.+8 u_{1} u_{2}^{3} \cos (\phi) \sin \left(\frac{\theta}{2}\right)^{2} \sin (\theta)+2 u_{1}^{2} u_{2}^{2}(2+\cos (2 \phi)) \sin (\theta)^{2}\right) \\
& +r\left(\mathrm{e}^{-\frac{1}{2}\left(u_{2}^{2}+\left(u_{1}-v_{1}\right)^{2}\right)}\left(1+r^{2}\right)+2 \mathrm{e}^{-u_{2}^{2}-\left(u_{1}-v_{1}\right)^{2}} r \cos (\eta)\right) \\
& \times\left(8 u_{1}^{2} v_{1}^{2} \cos \left(\frac{\theta}{2}\right)^{4} \cos (\eta)+8 u_{1} u_{2} v_{1}^{2} \cos \left(\frac{\theta}{2}\right)^{2} \cos (\eta+\phi) \sin (\theta)\right. \\
& \left.+2 u_{2}^{2} v_{1}^{2} \cos (\eta+2 \phi) \sin (\theta)^{2}\right) \\
& -\left(2\left(u_{1}^{2}+r^{2} v_{1}^{2}\right) \cos \left(\frac{\theta}{2}\right)^{2}+2 u_{2}^{2} \sin \left(\frac{\theta}{2}\right)^{2}+2 u_{1} u_{2} \cos (\phi) \sin (\theta)\right. \\
& \left.\left.+\frac{1}{2} \mathrm{e}^{-\frac{1}{2}\left(u_{2}^{2}+2\left(u_{1}-v_{1}\right)^{2}\right)} r\left(4 u_{1} v_{1} \cos \left(\frac{\theta}{2}\right)^{2} \cos (\eta)+2 u_{2} v_{1} \cos (\eta+\phi) \sin (\theta)\right)\right)^{2}\right] .
\end{aligned}
$$

The minimum values of this function with respect to $\theta$ and $\phi$ have been computed numerically and the results are shown in figure 5. Each plot in this figure contains two curves showing $Q(\rho)$ as a function of the relative phase $\eta$ corresponding to two different values of relative weight factor $r$. The amount of SPS varies with the relative phase in a similar way for all the plots. For all parameter values in all plots $Q(\rho) \leqslant 0$. This happens because the most general superposition of two two-mode coherent states can be transformed with the help of a $U(2)$ transformation into a product state with one factor being a coherent state, and the other a superposition of two one-mode coherent states

$\frac{1}{N}\left(\left|u_{1}^{\prime}\right\rangle+r \exp (\mathrm{i} \eta)\left|v_{1}^{\prime}\right\rangle\right)\left|v_{2}^{\prime}\right\rangle=\frac{1}{N} \mathcal{U}\left(S^{(c)}(U)\right)\left(\left|u_{1}, u_{2}\right\rangle+r \exp (\mathrm{i} \eta)\left|v_{1}, v_{2}\right\rangle\right)$.

Thus if $Q(\rho ; \alpha)$ turns out to be nowhere negative, the minimization chooses that $U(2)$ transformed mode which is in a coherent state.

It is interesting to point out that for a factorized two-mode state such as the expression on the left-hand side of equation (3.9), the mode $a(\bar{\alpha})$ which minimizes $Q(\rho ; \alpha)$ is generally neither of the two initial modes but a non-trivial combination of them.

\section{Concluding remarks}

The main aim of this paper has been to develop a specific signature of non-classicality for two-mode states. Both quadrature squeezing and SPS are well-defined concepts for a single mode. In this paper we have extended the notion of SPS to two modes by showing how to choose the appropriate single mode which shows SPS to the maximum extent, considering 

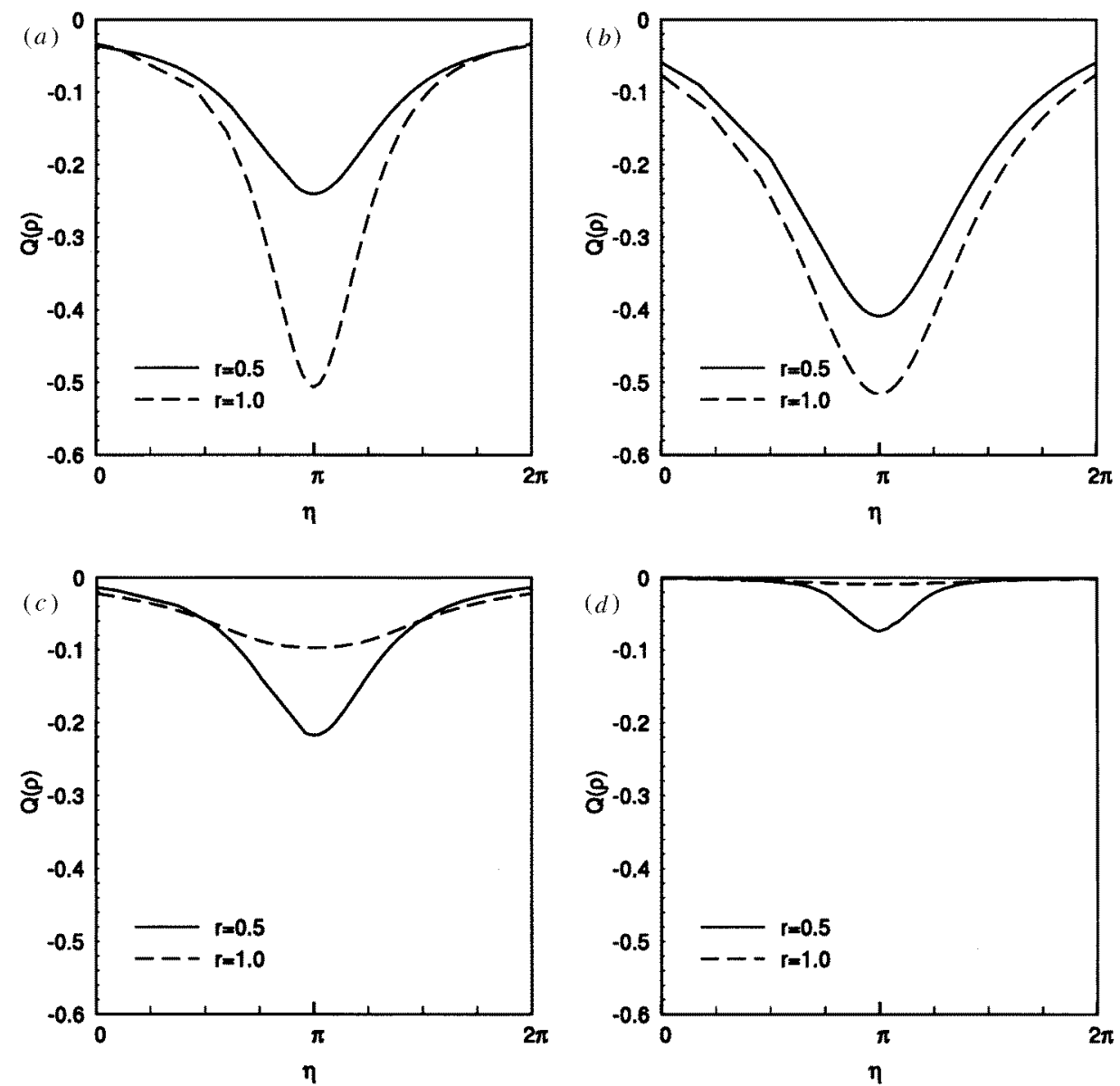

Figure 5. Plots of invariant Mandel parameter $Q(\rho)$ for superposition of two two-mode coherent states as a function of the phase difference between the two states are shown for two different values of relative weight, $r=0.5$ and $r=1.0$, and a given set of displacements. Values of displacements $u_{1}, u_{2}$ and $v_{2}$ are $(0.5,0.5,1.0),(0.5,1.0,1.0),(1.5,1.0,1.0)$ and $(1.5,1.0,0.5)$ for $(a)-(d)$, respectively.

all modes related to each other by passive $U(2)$ transformations as equivalent. A similar treatment of quadrature squeezing has been given elsewhere.

We would like to comment briefly on the role played by the choice of the denominator of $Q(\rho ; \alpha)$. Any choice which is everywhere non-negative will not change the qualitative results obtained from the minimization of $Q(\rho ; \alpha)$, i.e. the super- or sub-Poissonian nature of the state $\rho$. However, the extent of SPS, and the location of the most non-classical mode, depend upon the exact choice one makes for the denominator. To illustrate this point we choose the two-mode Fock state $\left|n_{1}, n_{2}\right\rangle$. The covariant Mandel parameter is given by

$$
\begin{gathered}
Q\left(n_{1}, n_{2}, \tilde{q}(\alpha)\right)=\frac{1}{4\left(n_{1}+n_{2}\right)}\left(-2\left(n_{1}+n_{2}\right)+\left(n_{1}+n_{2}\right)^{2}+\left(n_{1}\left(1-n_{1}\right)+n_{2}\left(1-n_{2}\right)\right)\right. \\
\left.\times\left(q_{1}^{2}+q_{2}^{2}\right)-2\left(n_{1}-n_{2}\right) q_{3}-\left(n_{1}+n_{2}\right)^{2} q_{3}^{2}\right) .
\end{gathered}
$$

This function reaches its minimum at $q_{3}=+1$ with minimum value $-\frac{n_{1}}{n_{1}+n_{2}}$ for $n_{1}>n_{2}$ 
and at $q_{3}=-1$ with the minimum value $-\frac{n_{2}}{n_{1}+n_{2}}$ for $n_{2}>n_{1}$. Thus for our $U(2)$ invariant choice of the denominator $\operatorname{Tr}\left(\rho \mathcal{A}^{\dagger} \mathcal{A}\right)$, for a Fock state, the mode with the larger number of photons is more non-classical. On the other hand if one chooses the $U(2)$ covariant denominator $\operatorname{Tr}\left(\rho a(\alpha)^{\dagger} a(\alpha)\right)$, for a Fock state, both the modes are equally non-classical irrespective of the number of photons present in each mode: the minimum value of this alternatively defined parameter is -1 for each mode. However, as explained in section 2 , the choice we have made for the denominator factor in $Q(\rho ; \alpha)$ seems more natural keeping in mind the kinematic aspects of the problem.

We may emphasize once again that quadrature squeezing and SPS are independent features of non-classicality, with the possibility in a given state $\rho$ of only one of them showing up and not the other. In that sense, therefore, the identification of a 'most nonclassical mode' definitely depends on whether one is looking at quadrature or amplitude squeezing, and in general the two may not agree at all. In any case, a complete treatment of the multimode quadrature squeezing problem, incorporating invariance under all passive mixing of modes, has been presented elsewhere. The main aim of the present investigation has been to attempt a treatment of amplitude squeezing in a similar spirit.

For one-mode fields the Mandel parameter can be written as a function of the number operator $a^{\dagger} a$ and hence is determined by (the moments of) the photon number distribution. In contrast, for two-mode fields the Mandel parameter for the $S U$ (2) transformed mode cannot be expressed as a function of the number operators $a_{1}^{\dagger} a_{1}$ and $a_{2}^{\dagger} a_{2}$ and therefore is not determined by the photon number distributions in the original modes. There could be other signatures of non-classicality which are meaningful at the one-mode level and can be extended in the spirit of this paper to more than one mode. In contrast, it will be interesting to explore the possibility of having signatures of non-classicality which are not definable at the one-mode level at all, but are present only at the two-mode level. These will be presented elsewhere.

\section{Appendix}

We give here the function $Q\left(z_{1}, z_{2}, a, b ; \alpha\right)$ for the squeezed coherent state with $z_{1}=u \mathrm{e}^{\mathrm{i} \varphi_{u}}$ and $z_{2}=v \mathrm{e}^{\mathrm{i} \varphi_{v}}$. The first term is the denominator, followed by the numerator terms arranged according to their dependence on $a$ and $b$. First the terms independent of $a, b$ appear, followed by the ones depending upon $a$ or $b$ alone, and then the ones depending on both $a$ and $b$. The last three terms originate from quadratic expressions of creation and annihilation operators and are not arranged.

$$
\begin{aligned}
Q\left(\alpha ; z_{1}, z_{2}, a,\right. & b)=2\left[-2+\cosh (2(a-b))+2 u^{2} \cosh (2(a-b))+\cosh (2(a+b))\right. \\
+ & 2 v^{2} \cosh (2(a+b))+2 u^{2} \cos \left(2 \varphi_{u}\right) \sinh (2(a-b)) \\
+ & \left.2 v^{2} \cos \left(2 \varphi_{v}\right) \sinh (2(a+b))\right]^{-1} \\
& {[} \\
\times & {\left[\frac{1}{8}\left(5+u^{4}+v^{4}+2\left(u^{2}+v^{2}\right)\right)+\frac{1}{8}\left(v^{4} \cos \left(4 \varphi_{v}\right)(-1+\cos (\theta))\right)\right.} \\
+ & \frac{1}{8}\left(\left(u^{2}-v^{2}\right)\left(2+u^{2}+v^{2}\right) \cos (\theta)\right)-\frac{1}{8}\left(u^{4} \cos \left(4 \varphi_{u}\right)(1+\cos (\theta))\right) \\
- & \frac{1}{16}\left(\left(1+u^{2}\left(2+u^{2}-u^{2} \cos \left(4 \varphi_{u}\right)\right)+v^{2}\left(2+v^{2}-v^{2} \cos \left(4 \varphi_{v}\right)\right)\right) \sin (\theta)^{2}\right)
\end{aligned}
$$




$$
\begin{aligned}
& +\frac{1}{4}\left(u v \cosh (2 a)\left(\begin{array}{c}
-8 \cos (\phi) \cos \left(\varphi_{u}-\varphi_{v}\right)-\left(2+u^{2}+v^{2}\right. \\
\left.+\left(u^{2}-v^{2}\right) \cos (\theta)\right) \sin (\phi) \sin \left(\varphi_{u}-\varphi_{v}\right) \\
-u^{2}(1+\cos (\theta)) \sin (\phi) \sin \left(3 \varphi_{u}+\varphi_{v}\right) \\
+v^{2}(1-\cos (\theta)) \sin (\phi) \sin \left(\varphi_{u}+3 \varphi_{v}\right)
\end{array}\right)\right) \sin (\theta) \\
& +\frac{1}{4}\left(\begin{array}{c}
u v\left(\begin{array}{c}
-8 \cos (\phi) \cos \left(\varphi_{u}+\varphi_{v}\right) \\
-\left(u^{2}-v^{2}+\left(2+u^{2}+v^{2}\right) \cos (\theta)\right) \\
\times \sin (\phi) \sin \left(\varphi_{u}+\varphi_{v}\right) \\
-v^{2}(1-\cos (\theta)) \sin (\phi) \sin \left(\varphi_{u}-3 \varphi_{v}\right) \\
-u^{2}(1+\cos (\theta)) \sin (\phi) \sin \left(3 \varphi_{u}-\varphi_{v}\right)
\end{array}\right) \sin (\theta) \sinh (2 a)
\end{array}\right) \\
& +\frac{1}{4}\left(u v \cosh (-2 b)\left(\begin{array}{c}
8 \sin (\phi) \sin \left(\varphi_{u}-\varphi_{v}\right) \sin (\theta) \\
+\cos (\phi) \cos \left(\varphi_{u}-\varphi_{v}\right) \\
\times\left(2+u^{2}+v^{2}+\left(u^{2}-v^{2}\right) \cos (\theta)\right) \\
-\cos (\phi) 2 u^{2} \cos \left(3 \varphi_{u}+\varphi_{v}\right) \cos \left(\frac{\theta}{2}\right)^{2} \\
-2 v^{2} \cos (\phi) \cos \left(\varphi_{u}+3 \varphi_{v}\right) \\
\times \sin \left(\frac{\theta}{2}\right)^{2} \sin (\theta)
\end{array}\right)\right) \\
& +\frac{1}{4}\left(u v\left(\begin{array}{c}
v^{2} \cos (\phi) \cos \left(\varphi_{u}-3 \varphi_{v}\right)(-1+\cos (\theta)) \\
+u^{2} \cos (\phi) \cos \left(3 \varphi_{u}-\varphi_{v}\right)(1+\cos (\theta)) \\
-\cos (\phi) \cos \left(\varphi_{u}+\varphi_{v}\right)\left(u^{2}-v^{2}\right. \\
\left.+\left(2+u^{2}+v^{2}\right) \cos (\theta)\right) \\
-8 \sin (\phi) \sin \left(\varphi_{u}+\varphi_{v}\right)
\end{array}\right) \sin (\theta) \sinh (-2 b)\right) \\
& +\frac{1}{8}\left(3+12 u^{2}+6 u^{4}+2 u^{4} \cos \left(4 \varphi_{u}\right)\right) \cos \left(\frac{\theta}{2}\right)^{4} \cosh (4(a-b)) \\
& +\frac{1}{8}\left(3+12 v^{2}+6 v^{4}+2 v^{4} \cos \left(4 \varphi_{v}\right)\right) \cosh (4(a+b)) \sin \left(\frac{\theta}{2}\right)^{4} \\
& +\frac{1}{2}\left(u^{2}\left(3+2 u^{2}\right) \cos \left(2 \varphi_{u}\right) \cos \left(\frac{\theta}{2}\right)^{4} \sinh (4(a-b))\right) \\
& +\frac{1}{2}\left(v^{2}\left(3+2 v^{2}\right) \cos \left(2 \varphi_{v}\right) \sin \left(\frac{\theta}{2}\right)^{4} \sinh (4(a+b))\right) \\
& +\cosh (2(a-b))\left(-\left(\left(1+2 u^{2}\right) \cos \left(\frac{\theta}{2}\right)^{2}\right)\right. \\
& \left.+\frac{u^{2} v^{2} \cos \left(2 \varphi_{u}\right) \sin (2 \phi) \sin \left(2 \varphi_{v}\right) \sin (\theta)^{2}}{2}\right) \\
& +\cosh (2(a-b))\left(-\left(\left(1+2 v^{2}\right) \sin \left(\frac{\theta}{2}\right)^{2}\right)\right. \\
& \left.-\frac{u^{2} v^{2} \cos \left(2 \varphi_{v}\right) \sin (2 \phi) \sin \left(2 \varphi_{u}\right) \sin (\theta)^{2}}{2}\right) \\
& +\left(-\left(u^{2} \cos \left(2 \varphi_{u}\right)(1+\cos (\theta))\right)+\frac{\left(1+2 u^{2}\right) v^{2} \sin (2 \phi) \sin \left(2 \varphi_{v}\right) \sin (\theta)^{2}}{4}\right) \\
& \times \sinh (2(a-b)) \\
& +\left(v^{2} \cos \left(2 \varphi_{v}\right)(-1+\cos (\theta))-\frac{u^{2}\left(1+2 v^{2}\right) \sin (2 \phi) \sin \left(2 \varphi_{u}\right) \sin (\theta)^{2}}{4}\right) \\
& \times \sinh (2(a-b)) \\
& +\frac{1}{4}\left(\left(\left(1+2 u^{2}\right)\left(1+2 v^{2}\right)+2 u^{2} v^{2} \cos (2 \phi) \cos \left(2 \varphi_{u}\right)\right.\right.
\end{aligned}
$$




$$
\begin{aligned}
& \left.\left.\times \cos \left(2 \varphi_{v}\right)\right) \cosh (2(a-b)) \cosh (2(a-b)) \sin (\theta)^{2}\right) \\
& +\frac{1}{8}\left(\left(4 u^{2}\left(1+2 v^{2}\right) \cos \left(2 \varphi_{u}\right)+2\left(1+2 u^{2}\right) v^{2} \cos (2 \phi) \cos \left(2 \varphi_{v}\right)\right)\right. \\
& \left.\times \cosh (2(a-b)) \sin (\theta)^{2} \sinh (2(a-b))\right) \\
& +\frac{1}{8}\left(\left(\left(1+2 u^{2}\right)\left(1+2 v^{2}\right) \cos (2 \phi)+8 u^{2} v^{2} \cos \left(2 \varphi_{u}\right) \cos \left(2 \varphi_{v}\right)\right)\right. \\
& \left.\times \sin (\theta)^{2} \sinh (2(a-b)) \sinh (2(a-b))\right) \\
& +\frac{1}{8}\left(\left(2 u^{2}\left(1+2 v^{2}\right) \cos (2 \phi) \cos \left(2 \varphi_{u}\right)+4\left(1+2 u^{2}\right) v^{2} \cos \left(2 \varphi_{v}\right)\right)\right. \\
& \left.\times \cosh (2(a-b)) \sin (\theta)^{2} \sinh (2(a-b))\right) \\
& +\frac{1}{2}\left(u v \cos (\phi)\left(3\left(1+u^{2}\right) \cos \left(\varphi_{u}-\varphi_{v}\right)+u^{2} \cos \left(3 \varphi_{u}+\varphi_{v}\right)\right)\right. \\
& \left.\times \cos \left(\frac{\theta}{2}\right)^{2} \cosh (2(2 a-b)) \sin (\theta)\right) \\
& +\frac{1}{2}\left(u v \cos (\phi)\left(u^{2} \cos \left(3 \varphi_{u}-\varphi_{v}\right)+3\left(1+u^{2}\right) \cos \left(\varphi_{u}+\varphi_{v}\right)\right)\right. \\
& \left.\times \cos \left(\frac{\theta}{2}\right)^{2} \sin (\theta) \sinh (2(2 a-b))\right) \\
& +\frac{1}{2}\left(u v \cos (\phi)\left(3\left(1+v^{2}\right) \cos \left(\varphi_{u}-\varphi_{v}\right)+v^{2} \cos \left(\varphi_{u}+3 \varphi_{v}\right)\right)\right. \\
& \left.\times \cosh (2(2 a+b)) \sin \left(\frac{\theta}{2}\right)^{2} \sin (\theta)\right) \\
& +\frac{1}{2}\left(u v \cos (\phi)\left(v^{2} \cos \left(\varphi_{u}-3 \varphi_{v}\right)+3\left(1+v^{2}\right) \cos \left(\varphi_{u}+\varphi_{v}\right)\right)\right. \\
& \left.\times \sin \left(\frac{\theta}{2}\right)^{2} \sin (\theta) \sinh (2(2 a+b))\right) \\
& +\frac{1}{2}\left(u v \operatorname { c o s } ( \frac { \theta } { 2 } ) ^ { 2 } \operatorname { s i n } ( \phi ) \left(-\left(u^{2} \sin \left(3 \varphi_{u}-\varphi_{v}\right)\right)\right.\right. \\
& \left.\left.+3\left(1+u^{2}\right) \sin \left(\varphi_{u}+\varphi_{v}\right)\right) \sin (\theta) \sinh (2(a-2 b))\right) \\
& +\frac{1}{2}\left(u v \sin (\phi)\left(v^{2} \sin \left(\varphi_{u}-3 \varphi_{v}\right)+3\left(1+v^{2}\right) \sin \left(\varphi_{u}+\varphi_{v}\right)\right)\right. \\
& \left.\times \sin \left(\frac{\theta}{2}\right)^{2} \sin (\theta) \sinh (-2(a+2 b))\right) \\
& -\frac{1}{2}\left(u v \operatorname { c o s h } ( - 2 ( a + 2 b ) ) \operatorname { s i n } ( \phi ) \left(3\left(1+v^{2}\right) \sin \left(\varphi_{u}-\varphi_{v}\right)\right.\right. \\
& \left.\left.+v^{2} \sin \left(\varphi_{u}+3 \varphi_{v}\right)\right) \sin \left(\frac{\theta}{2}\right)^{2} \sin (\theta)\right) \\
& +\frac{1}{2}\left(u v \cos \left(\frac{\theta}{2}\right)^{2} \cosh (2(a-2 b)) \sin (\phi)\right. \\
& \left.\times\left(-3\left(1+u^{2}\right) \sin \left(\varphi_{u}-\varphi_{v}\right)+u^{2} \sin \left(3 \varphi_{u}+\varphi_{v}\right)\right) \sin (\theta)\right)
\end{aligned}
$$




$$
\begin{aligned}
& +\frac{1}{2}\left(u^{2} v^{2} \cos (2 \phi) \sin \left(2 \varphi_{u}\right) \sin \left(2 \varphi_{v}\right) \sin (\theta)^{2}\right)-\left[-\frac{1}{2}\right. \\
& \left.+\frac{(1+\cos (\theta))\left(\left(1+2 u^{2}\right) \cosh (2(a-b))+2 u^{2} \cos \left(2 \varphi_{u}\right) \sinh (2(a-b))\right)}{4}\right] \\
& +\frac{(1-\cos (\theta))\left(\left(1+2 v^{2}\right) \cosh (2(a-b))+2 v^{2} \cos \left(2 \varphi_{v}\right) \sinh (2(a-b))\right)}{4} \\
& \left.+u v \sin (\theta)\left(\begin{array}{c}
\sin (\phi)\left(\cosh (-2 b) \sin \left(\varphi_{u}-\varphi_{v}\right)\right. \\
\left.-\sin \left(\varphi_{u}+\varphi_{v}\right) \sinh (-2 b)\right) \\
+\cos (\phi)\left(\cos \left(\varphi_{u}-\varphi_{v}\right) \cosh (2 a)\right. \\
\left.+\cos \left(\varphi_{u}+\varphi_{v}\right) \sinh (2 a)\right)
\end{array}\right]^{2}\right] .
\end{aligned}
$$

\section{Acknowledgment}

BA thanks University Grants Commission India for financial support.

\section{References}

[1] Teich M C and Saleh B E A 1988 Progress in Optics vol 26, ed E Wolf (Amsterdam: North-Holland) Walls D F 1979 Nature 280451

[2] Stoler D 1970 Phys. Rev. D 13217

Yuen H P 1976 Phys. Rev. A 132226

Walls D F 1983 Nature 306141

[3] Kimble H J, Dagenais M and Mandel L 1977 Phys. Rev. Lett. 39691

[4] Arvind B, Dutta B, Simon R and Mukunda N 1995 Phys. Rev. A 521609

[5] Simon R, Mukunda N and Dutta B 1994 Phys. Rev. A 491567

[6] Mandel L 1979 Opt. Lett. 4205

[7] Short R and Mandel L 1983 Phys. Rev. Lett. 51384

[8] Agarwal G S 1986 Phys. Rev. Lett. 57827

[9] Lee C T 1990 Phys. Rev. A 421608

[10] Jordan T F 1974 Linear Operators in Quantum Mechanics (New York: Wiley) Lion G and Vergne M 1980 The Weil Representation, Maslov Index, and Theta series (Basel: Birkhauser)

[11] Klauder J R and Sudarshan E C G 1968 Fundamentals of Quantum Optics (New York: Benjamin)

[12] Yurke B, McCall S L and Klauder J R 1986 Phys. Rev. A 334033

Simon R and Mukunda N 1990 Phys. Lett. 143A 165, also [4] above 